\title{
Cannabis-Induced Mania Following COVID-I9 Self-Medication: A Wake-Up Call to Improve Community Awareness
}

This article was published in the following Dove Press journal: International Medical Case Reports Journal

\author{
Mark Mohan Kaggwa (D) \\ Felix Bongomin (iD) ${ }^{2}$ \\ Sarah Maria Najjuka $\mathbb{D D}^{3}$ \\ Godfrey Zari Rukundo' \\ Scholastic Ashaba' \\ 'Department of Psychiatry, Faculty of \\ Medicine, Mbarara University of Science \\ and Technology, Mbarara, Uganda; \\ ${ }^{2}$ Department of Medical Microbiology \\ and Immunology, Faculty of Medicine, \\ Gulu University, Gulu, Uganda; ${ }^{3}$ College \\ of Health Sciences, Makerere University, \\ Kampala, Uganda
}

Context: Self-medication is becoming common during the coronavirus disease -2019 (COVID-19) pandemic due to the increasing popularity of home-based management of asymptomatic and mild cases. In this case report, we describe a patient who developed manic symptoms as a result of self-medication with a regimen containing cannabis to manage COVID-19 symptoms.

Case Details: A 52-year-old man with no prior history of a mental disorder, presented with a one-week history of talking more than usual, poor sleep, destructiveness, irritability, and altered mental status, following use of homemade remedies containing oranges, garlic, ginger, onions, honey, lemon, and cannabis to treat COVID-19 related symptoms over a 2-week period. This was his index presentation with such symptoms in his life. He had never used any substance of addiction before, did not have any known chronic medical condition, and had no family member with a history of any known mental illness. He was a suspect because his father had tested positive for COVID-19 and was undergoing treatment. He tested negative for COVID-19 after 3-weeks of initial COVID-19 like symptoms, urine sample was positive for tetrahydrocannabinol (THC), and he had normal investigations. He was managed with a mood stabilizer (oral carbamazepine at a dose of $200 \mathrm{mg}$ three times daily), antipsychotic (chlorpromazine $200 \mathrm{mg}$ twice daily), a sedative (diazepam 10mg at before bedtime), and occupational therapy. All manic symptoms resolved in a period of two weeks.

Conclusion: Cannabis induced mental illness following self-medication for COVID-19 like symptoms is on the rise in the population. Due to increasing COVID-19 cases globally, hospital congestion, the popularity of home-based care guidelines for asymptomatic and mild COVID-19 to reduce hospital burden in many countries, and easy access to cannabis. With no approved cure for COVID-19, patients are turning to natural remedies to relieve symptoms of COVID-19. Emphasis on prevention of this insalubrious self-medication among the COVID-19 patients is needed to stop complication related to cannabis use.

Keywords: COVID-19, cannabis, mania, bipolar, mental disorder, self-medication, cannabis-induced mania, homemade remedies for COVID-19, cannabis use complication

\section{Introduction}

Cannabis-induced mania follows recent use or intoxication with or withdrawal from cannabis. ${ }^{1}$ The patient develops clinically significant behavioral and psychological changes that meet criteria for manic episode including euphoria, expansive, or irritable mood, grandiose delusions, decreased need for sleep, more talkative than usual, flight of ideas, and increased goal-directed ideas. ${ }^{1}$ Cannabis components such
Correspondence: Felix Bongomin Department of Medical Microbiology and Immunology, Faculty of Medicine, Gulu University, P. O. Box 166, Gulu, Uganda Tel +256-78452-3395

Email drbongomin@gmail.com
International Medical Case Reports Journal 2021:14 |2|-125 
as delta-9-tetrahydrocannabinol (delta-9-THC) have high potency for G-protein coupled cannabinoid receptors $\mathrm{CBl}$ and $\mathrm{CB} 2$, responsible for the symptomatology of the induced mania, but the mechanism is not well documented. ${ }^{1,2}$

Cannabis via its receptors has a mechanistic role on memory, appetite, and stress response (anxiety, mood disturbance, and sleep disturbance), hence its continuous use to treat or control symptoms of the different medical conditions by physician. ${ }^{3}$ Cannabis especially its synthetic derivative delta-9-THC has been approved in the management of nausea and vomiting caused by chemotherapy, for anorexia, spasticity in multiple sclerosis, seizure reduction in rare forms of epilepsy, and weight loss in individuals with AIDS. ${ }^{1,4}$

As the search for the therapeutic armamentarium for the coronavirus disease - 2019 (COVID-19) continues, recent studies have reported the importance of cannabis in managing COVID-19 related physical and mental symptoms. $^{4-9}$ The potential effectiveness of cannabis in managing COVID-19 symptoms has made many COVID19 patients self-medicate. ${ }^{10}$ Self-medication with home remedies containing cannabis possesses a risk due to cannabis use induced mental illness such as psychosis, mania, or depression. The use of cannabis is also associated with substance use disorder, brain damage, cardiovascular disorders, and severe mental illness. ${ }^{11-13}$

The following case illustrates a suspected case of COVID-19 who self-medicated with home remedies containing cannabis and ended up developing severe manic symptoms requiring hospitalization. As per our literature search in various databases (Google scholar, PubMed, and Scopus), this is the first case of cannabis-induced mania as a result of self-medication to prevent COVID-19.

\section{Case Report}

A 52-year-old married Ugandan man, was involuntarily admitted to a psychiatry unit of a tertiary referral hospital in Uganda with concerns of one-week history of persistent talking, poor sleep, destructiveness, irritability, and altered mental status. This behavior followed 2-weeks use of homemade remedies containing cannabis to treat COVID-19 related symptoms.

Three-weeks prior to his admission, he travelled with his father for a burial. His father contracted COVID-19, developed symptoms, and was taken for treatment at the national referral hospital after investigations confirmed he had COVID-19. As his father was undergoing treatment; He started experiencing COVID-19 like symptoms, including difficulty in breathing, nasal blockage, high-grade fever, excessive sweating, headache, lack of smell, general body weakness, and cough. His relative advised him to selfmedicate. He self-medicated with a home remedy containing oranges, garlic, ginger, onions, honey, lemon, and cannabis. He would do herbal steaming three times a day with hot water having lemon leaves, cannabis, a Menthol containing "dragon," and later drunk the solution. He could also take toff plus (Caffeine, Chlorpheniramine, Paracetamol and Phenylpropanolamine combination) medication and Azithromycin twice a day. He used the green leaves of cannabis freshly harvested from a neighbors' garden, who planted them when the cannabis effect on COVID-19 started circulating and preached earlier in the pandemic. After using the remedy, he would get relief from the COVID-19 like symptoms and felt happier. His heart would beat faster than usual and immediately, felt hungry, thus ending up eating more amounts than usual and becoming more physically active than usual. His relatives and wife noticed he had become more demanding than usual, mostly using his position as the firstborn; his demands to be respected, or it would storm into a heated augment.

Two weeks later, immediately after his father's discharge, he started asking him and siblings to give him money and a car to go for COVID-19 testing and treatment. When they tried assuring him that he may not be positive despite his symptoms; he became aggressive, attacked the father and vandalized his car. Before this abnormal behavior, he had sleepless nights trying to find ways to treat his symptoms. He was more talkative than usual, especially about his successful young siblings and father who refuse to help him because he is poor, despite him helping them with school fees during their studies. He was also more prayerful than usual and was putting much emphasis in preparing to go to heaven. During this week of mental disturbance, he was incapable of accomplishing any of his daily activities, such as digging. He recklessly spent all the small savings on buying items he did not need. He also gave out some of his possession to strangers he considered needy an act unusual of him.

This was his index presentation of such symptoms in his life. He had never used any substance of abuse before, did not have any known chronic medical condition, and had no family member with a history of manic, depressive symptoms, or other mental illness. The patient had no prior injury to the head or being involved in severe trauma, having an episode with loss of consciousness, or seizure. 
Apart from COVID-19 related stressor in his life, there was no history of any known stressor. Despite having COVID-19 like symptoms, he did not have any COVID19 neurological symptoms, such as seizures, loss of consciousness, neck stiffness, visual impairment, however, he had a severe headache and dizziness.

At admission, he was dyspneic with a respiratory rate of 29 breaths per minute, afebrile $\left(36.3^{\circ} \mathrm{c}\right)$, blood pressure $113 / 86 \mathrm{mmHg}$, pulse rate 89 beats per minute, oxygen saturation of $93 \%$ on room air, tremorous, sweating, and restless. The chest was clear, with normal breath sounds. He had an irritable mood, grandiose delusions, poor concentration, very poor insight, but no hallucinations. Other system examinations were unremarkable.

The baseline investigations included: a complete blood count, renal functional tests, liver-functional tests, human immunodeficiency virus (HIV) serology, urinalysis, head and chest computerized tomography (CT) scan which were normal. A 10-panel urine drug screen was done and was positive for tetrahydrocannabinol (THC) alone. A sample for COVID-19 test was taken off and turned out negative.

Based on the above, a diagnosis of cannabis-induced mania was made. He was tranquillized with intramuscular chlorpromazine $200 \mathrm{mg}$ and intravenous diazepam $20 \mathrm{mg}$ as stat doses. He was maintained on oral carbamazepine at a dose of $200 \mathrm{mg}$ three times daily, chlorpromazine $200 \mathrm{mg}$ twice daily, and diazepam $10 \mathrm{mg}$ at before bedtime. Due to the excessive energy, he had, we involved the occupational therapy team that engaged him in physical activities for energy diversion. He was kept isolated from other patients for the first few days until his COVID-19 results were available.

Within 2-weeks of hospitalization, all symptoms had resolved. By discharge, he had achieved substantial insight about the condition and its etiology. On the subsequent monthly reviews, he had no active symptoms.

\section{Discussion}

The number of COVID-19 cases is increasing globally with over 86,000,000 cases and 1,900,000 deaths in $2020 .{ }^{14}$ As hospitals are overwhelmed with the numbers of cases, home-based management have been encouraged for asymptomatic individuals or those with mild symptoms. ${ }^{15,16}$ This has lead lack of regular health services such as psychiatric services that during the pandemic and many of the patients have turned to self-medication to treat both physical and psychiatry symptoms (sleep, anxiety, depression, and stress). ${ }^{17}$ With a lot of information circulating about possible remedies, including the use of cannabis, the complications of these self-medicated regimens, such as mental illness, metabolic disorders, and cardiovascular diseases, may increase within the community. ${ }^{18}$ The present case describes a mental illness (mania) complication following self-medication with cannabis containing regimen in a patient with no previous history of mood symptoms (hypomania or depression), personal history of mental illness, or family history of mental illness. These characteristics are different from the common manic episodes reported in previous studies. ${ }^{19}$ The age of onset of manic symptoms coincides with the bimodal distribution on the incidence of mania, between 15 and 24 years and 45 and54 years, and cannabis use being a trigger. ${ }^{19-22}$

Following positive tetrahydrocannabinol (THC) in urine, and no other component in the regimen can induce mania; the diagnosis of cannabis-induced mania was made given presence of cannabis intoxication symptoms (increased appetite, tachycardia, and euphoria) during the early symptom presentation and withdraw symptoms (irritability, sleep difficulties, restlessness, tremorous, sweating, fever, and headache) during the admission period. ${ }^{1}$ Thus, this was considered to be unlikely late-onset Bipolar Affective disorder (BAD) because of no previous risk factors for BAD, such as mood episodes, family history, or cluster B personality traits. Other differential diagnosis included a hyperactive type of delirium, from possible COVID-19 infection or CNS manifestation of COVID-19 because the patient had COVID-19 like symptoms. Despite testing negative for COVID-19, 3-weeks after the onset of symptoms, a period long enough for recovery from the infection, the suspicion of a hyperactive type of delirium was unlikely, all baseline investigations being unremarkable. Adjustment disorder with manic symptoms is another possible differential diagnosis due to having contact with a COVID-19 patient who was a close family member. However, the presence of cannabis intoxication and withdrawal symptoms, make it less likely.

Various complications are associated with a diagnosis of mania in COVID-19 patients with cannabis due to the augmented CNS inflammation hence atrophy leading to long-term severe mental illness. ${ }^{23}$ The use of cannabis has hazardous effects when combined with some psychoactive drugs that would have managed the mental symptoms, through the saturation of the cytochrome P450 enzyme, increasing plasma concentration and or toxicity of some drugs such as phenytoin. ${ }^{24}$ The patients 
may also end up continuously using cannabis due to its addictive properties which may lead to increased mental illness burden as the second predicted pandemic of mental illness. ${ }^{25}$ This unmonitored use of cannabis leads to several other complications in the general population, including multi-substance use disorder, brain damage, cardiovascular diseases, and severe mental illness. ${ }^{11-13}$ Effective management of the manic symptoms and the complication of cannabis use is needed; for better quality life among patients affected with COVID-19.

Similar to our patient management, cannabis-induced mania management involves the use of mood stabilizers for the manic symptoms and benzodiazepines for agitation and anxiety symptoms, in case of psychotic phenomena antipsychotics are also recommended. ${ }^{26}$ With the surge of mental illness during the pandemic, ${ }^{17}$ internet best interventions and telepsychiatry are recommended to manage psychiatry symptoms. ${ }^{27,28}$ Internet cognitive behavior therapy a pandemic cost-effective, cognitive behavioral method for managing mental symptoms insomnia, delusions, and other manic related symptoms, may be a preferred alternative for many patients in an era of reducing COVID-19 spread. $^{29,30}$

The use of cannabis in management of COVID-19 symptoms may be on rise because of the various mechanisms proposed for its effectiveness towards COVID-19. These mechanism include: 1) hypotensive properties through renin angiotensin system modeling, ${ }^{4} 2$ ) inhibiting the Angiotensin Converting Enzyme receptors 2, receptors involved in virus entry into the respiratory system, ${ }^{4} 3$ ) suppression of the immune and inflammation functions because COVID-19 induces cytokine storm, ${ }^{5-8} 4$ ) agonist to peroxisome proliferator-activated receptor gamma (PPAR $\gamma$ ), thus antiviral properties, ${ }^{6} 5$ ) regulators of fibroblast/myofibroblast activation through PPAR $\gamma$, thus inhibiting the pulmonary fibrosis development in recovered patients (ameliorating lung function), ${ }^{6}$ and 6) serotonin and dopamine neurotransmitters regulation hence treating the mental illness related symptoms such as depression, stress, and anxiety. ${ }^{4}$ Despite its potential effectiveness, it increases the risk for mental illness symptoms as illustrated by the current case especially with the increasing promoted self-medication approach through home-based management using natural remedies.

\section{Conclusions}

Cannabis induced mental illness following self-medication for COVID-19 like symptoms is on the rise in the population due to increasing cases of COVID-19 cases globally and easy access to cannabis in many countries. The use cannabis to treat COVID-19 may be on the rise due to home-based care guidelines being encouraged for asymptomatic and mild cases of COVID-19 to reduce congestion in the hospitals which are already burdened by higher numbers. Moreover, most people are turning to natural remedies to relieve symptoms of COVID-19 since the disease has no approved cure. Emphasis on prevention of this insalubrious self-medication among the COVID-19 patients is needed to stop complication related to cannabis use.

\section{Ethics}

Institutional approval was not required to publish this case. However, after recovery, the patient provided a written informed consent to have the case published and notify the community about complications of self-medications for COVID-19 symptoms.

\section{Acknowledgment}

The authors acknowledge the patient for pushing so much to have this case published to make the community aware.

\section{Author Contributions}

All authors made a significant contribution to the work reported, be it in the conception, study design, execution, acquisition of data, analysis and interpretation, or in all these areas; took part in drafting, revising, or critically reviewing the article; gave final approval of the version to be published; have agreed on the journal to which the article has been submitted; and agree to be accountable for all aspects of the work.

\section{Funding}

Felix Bongomin's Research work was supported by the Fogarty International Center of the National Institutes of Health, US Department of State's Office of the US Global AIDS Coordinator and Health Diplomacy (S/GAC), and President's Emergency Plan for AIDS Relief (PEPFAR) under Award Number 1R25TW011213. The content is solely the responsibility of the authors and does not necessarily represent the official views of the National Institutes of Health.

\section{Disclosure}

The authors declare that they have no conflicts of interest for this work. 


\section{References}

1. Arlington APA. Diagnostic and Statistical Manual of Mental Disorders. J Am Psychiatric Assoc. 2013:21.

2. Sideli L, Quigley H, La Cascia C, Murray R. Cannabis use and the risk for psychosis and affective disorders. J Dual Diagnosis. 2020;16 (1):22-42. doi:10.1080/15504263.2019.1674991

3. Rong C, Lee Y, Carmona NE, et al. Cannabidiol in medical marijuana: research vistas and potential opportunities. Pharmacol Res. 2017;121:213-218. doi:10.1016/j.phrs.2017.05.005

4. O'Sullivan SE, Stevenson CW, Laviolette SRJC, Research C. Could Cannabidiol Be a Treatment for Coronavirus Disease-19-Related Anxiety Disorders? Cannabis Cannabinoid Res. 2020.

5. Costiniuk CT, Jenabian M-AJC, Reviews GF. Acute inflammation and pathogenesis of SARS-CoV-2 infection: cannabidiol as a potential anti-inflammatory treatment? Growth Factor Rev. 2020;53:63-65. doi:10.1016/j.cytogfr.2020.05.008

6. Esposito G, Pesce M, Seguella L, et al. The potential of cannabidiol in the COVID-19 pandemic: a hypothesis letter. $\mathrm{Br} J$ Pharmacol. 2020;177(21):4967.

7. Onaivi ES, Sharma V. Cannabis for COVID-19: can cannabinoids quell the cytokine storm? Future Sci. 2020;6(8):FSO625. doi:10.2144/fsoa-2020-0124

8. Rossi F, Tortora C, Argenziano M, Di Paola A, Punzo F. Cannabinoid Receptor Type 2: a Possible Target in SARS-CoV-2 (CoV-19) Infection? Int J Mol Sci. 2020;21(11):3809.

9. Xiong J, Lipsitz O, Nasri F, et al. Impact of COVID-19 pandemic on mental health in the general population: a systematic review. $J$ Affect dis. 2020;277:55-64.

10. Molento MB. COVID-19 and the rush for self-medication and self-dosing with ivermectin: a word of caution. One Health. 2020 10:100148.

11. Jouanjus E, Lapeyre-Mestre M, Micallef J, Abuse F. Cannabis use: signal of increasing risk of serious cardiovascular disorders. $J \mathrm{Am}$ Heart Assoc. 2014;3(2):e000638. doi:10.1161/JAHA.113.000638

12. Karila L, Roux P, Rolland B, et al. Acute and long-term effects of cannabis use: a review. Curr Pharm Design. 2014;20(25):4112-4118. doi:10.2174/13816128113199990620

13. Coscas S, Benyamina A, Reynaud M, Karila L. Psychiatric complications of cannabis use. La Revue du praticien. 2013;63 (10):1426-1428.

14. Worldometer. Coronavirus Death Toll and Trends - worldometer; 2021. Available from: https://www.worldometers.info/coronavirus/ coronavirus-death-toll/. Accessed January 1, 2021.

15. Thota SM, Balan V, Sivaramakrishnan VJPR. Natural products as home-based prophylactic and symptom management agents in the setting of COVID. Phytotherapy Res. 2020;34(12):3148-3167. doi:10.1002/ptr.6794

16. Ministry of Health Uganda; The Department of Health Promotion EHC. Basic information for covid-19 patients undergoing home based isolation and care; 2020. Available from: https://www.health. go.ug/cause/basic-information-for-covid-19-patients-undergoinghome-based-isolation-and-care/. Accessed February 24, 2021.
17. Hao F, Tan W, Jiang L, et al. Do psychiatric patients experience more psychiatric symptoms during COVID-19 pandemic and lockdown? Brain. 2020;87:100-106.

18. Suchithra B, As NU. Study on Awareness about Self-Medications and it's Complications Among Homemakers. J Health Allied Sci. 2017;7 (01):061-065.

19. Rowland TA, Marwaha S. Epidemiology and risk factors for bipolar disorder. Therapeutic Adv Psychopharmacol. 2018;8(9):251-269. doi:10.1177/2045125318769235

20. Kroon JS, Wohlfarth TD, Dieleman J, et al. Incidence rates and risk factors of bipolar disorder in the general population: a populationbased cohort study. Bipolar Disorders. 2013;15(3):306-313. doi:10.1111/bdi.12058

21. Gibbs M, Winsper C, Marwaha S, Gilbert E, Broome M, Singh S. Cannabis use and mania symptoms: a systematic review and meta-analysis. $J$ Affect Dis. 2015;171:39-47. doi:10.1016/j. jad.2014.09.016

22. Bally N, Zullino D, Aubry J-M. Cannabis Use and First Manic Episode. J AffecT Dis. 2014;165:103-108.

23. Steardo Jr L, Steardo L, Verkhratsky A. Psychiatric face of COVID-19. Translat Psychiatry. 2020;10(1):1-12.

24. Rong C, Carmona NE, Lee YL, et al. Drug-drug interactions as a result of co-administering $\triangle 9$-THC and CBD with other psychotropic agents. Exp Opinion Drug Safety. 2018;17(1):51-54. doi:10.1080/14740338.2017.1397128

25. Choi KR, Heilemann MV, Fauer A, Mead M. A second pandemic: mental health spillover from the novel coronavirus (COVID-19). $J$ Am Psychiatric Nurs Assoc. 2020;26(4)340-343.

26. Kondo KK, Morasco BJ, Nugent SM, et al. Pharmacotherapy for the treatment of cannabis use disorder: a systematic review. Ann Int Med. 2020;172(6):398-412. doi:10.7326/M19-1105

27. Burgess C, Miller CJ, Franz A, et al. Practical lessons learned for assessing and treating bipolar disorder via telehealth modalities during the COVID-19 pandemic. Bipolar Disorders. 2020;22 (6):556-557. doi:10.1111/bdi.12969

28. Ho CS, Chee CY, Ho RC. Mental health strategies to combat the psychological impact of COVID-19 beyond paranoia and panic. Ann Acad Med Singapore. 2020;49(3):155-160.

29. Zhang M, Ho RJT. Moodle: The Cost Effective Solution for Internet Cognitive Behavioral Therapy (I-CBT) Interventions. Tech Health Care. 2017;25(1):163-165.

30. Soh HL, Ho RC, Ho CS, Tam WWJSM. Efficacy of digital cognitive behavioural therapy for insomnia: a meta-analysis of randomised controlled trials. Sleep Med. 2020;75:315-325. doi:10.1016/j. sleep.2020.08.020
International Medical Case Reports Journal

\section{Publish your work in this journal}

The International Medical Case Reports Journal is an international, peer-reviewed open-access journal publishing original case reports from all medical specialties. Previously unpublished medical posters are also accepted relating to any area of clinical or preclinical science. Submissions should not normally exceed 2,000 words or 4 published pages including figures, diagrams and references. The manuscript management system is completely online and includes a very quick and fair peer-review system, which is all easy to use. Visit http://www.dovepress.com/testimonials.php to read real quotes from published authors. 